\title{
Surgical correction of ptosis of the eyebrow
}

\author{
F. P. ENGLISH AND N. D. McDERMOTT \\ Department of Ophthalmology, Mater Hospital, University of Queensland, Australia
}

In recent years there has been a reappraisal of the surgical principles concerned in the management of the ophthalmic complications of facial nerve paralysis. This is especially mirrored in the utilization of the palpebral spring (Morel-Fatio and Lalardrie, 1967) and of silicone cerclage (Arion, 1972) in an attempt to produce dynamic restoration of lid function. A more vigorous approach is also advocated to correct the eyebrow defect when this occurs in facial palsy.

Ptosis of the eyebrow is an uncommon but disabling condition generally representative of severe damage to the facial nerve. Such injury has been observed subsequent to unavoidable trauma in the surgery of acoustic nerve neuroma. The infrequency of this condition in facial paralysis probably correlates with the specialized innervation of the upper face.

Characteristically the affected brow droops excessively so that the hair line is much lower than its fellow. The ptotic element is most conspicuous in the temporal half of the eyebrow, and can be so manifest as to obscure vision and cause marked encroachment on the visual field (Fig. I). The disfiguring situation is readily rectified by plastic surgery restoring vision and producing a cosmetically acceptable appearance.

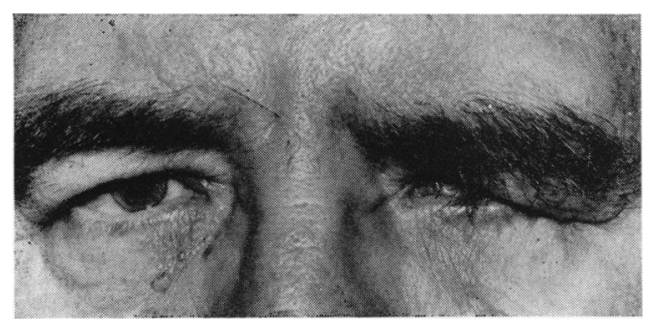

FIG. I Preoperative photograph, showing ptosis of the eyebrow

\section{Surgical procedure}

Under general anaesthesia a curved full-thickness linear incision is made immediately above the brow hair line. Traction with skin hooks inserted in the freed brow allows this to be aligned with the normal opposite hair line.

The resultant overlap produced by these manipulations is marked with a skin pen or pencil (Fig. 2, overleaf) and an incision is made along this line of demarcation. The ellipse of skin and orbicularis oculi is undermined and dissected clear, attention being paid to absolute haemostasis. Interrupted $5^{-0}$ silk mattress sutures are inserted and traction applied, thus elevating the affected eyebrow to its correct height. Alterations may be performed at this stage, as occasionally the temporal section requires a wider excision. Sutures are tied and a Telfa dressing and light pressure pad applied. 
(2)

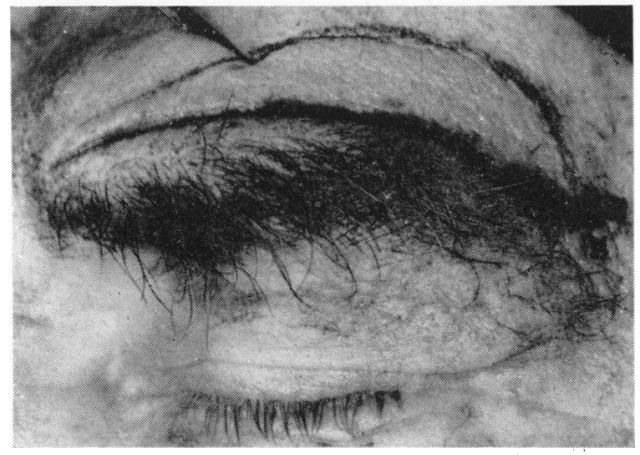

(3)

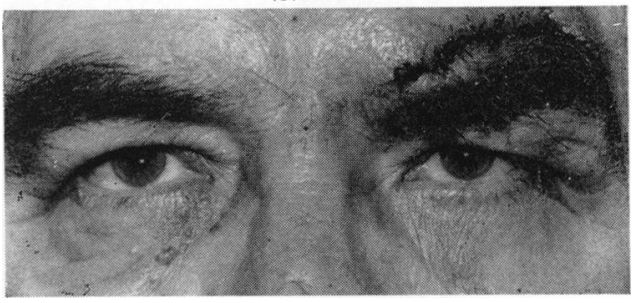

FIG. 2 Outlining the upper border of the area to be excised

FIG. 3 Appearance 24 hrs after operation demonstrating restoration of normal brow height

\section{Conclusion}

This simple technique allows for correction of the ptotic eyebrow, restoring normal function and ensuing good cosmesis (Fig. 3).

\section{References}

ARION, H. G. (1972) Int. Surg., 57, 48

MOREL-Fatio, D., and LALARDRIE, J. P. (I967) In "Proc. II Int. Symp. on Plastic and Reconstructive Surgery of the Eye and Adnexa", ed. B. Smith and J. M. Converse, p. 38o. Mosby, St. Louis 